\title{
Transconjuctival Incision with Lateral Paracanthal Extension for Corrective Osteotomy of Malunioned Zygoma
}

\author{
Jae-Ho Chung ${ }^{1}$, \\ $\mathrm{Hi}$-Jin You', \\ Na-Hyun Hwang ${ }^{2}$, \\ Deok-Woo Kim ${ }^{1}$, \\ Eul-Sik Yoon ${ }^{2}$ \\ ${ }^{1}$ Department of Plastic and Reconstructive \\ Surgery, Korea University Ansan Hospital, \\ Ansan; \\ ${ }^{2}$ Department of Plastic and Reconstructive \\ Surgery, Korea University Anam Hospital, \\ Korea University College of Medicine, Anam, \\ Korea
}

No potential conflict of interest relevant to this article was reported.

\begin{abstract}
Background: Conventional correction of malunioned zygoma requires complete regional exposure through a bicoronal flap combined with a lower eyelid incision and an upper buccal sulcus incision. However, there are many potential complications following bicoronal incisions, such as infection, hematoma, alopecia, scarring and nerve injury. We have adopted a zygomaticofrontal suture osteotomy technique using transconjunctival incision with lateral paracanthal extension. We performed a retrospective review of clinical cases underwent correction of malunioned zygoma with the approach to evaluate outcomes following this method.

Methods: Between June 2009 and September 2015, corrective osteotomies were performed in 14 patients with malunioned zygoma by a single surgeon. All 14 patients received both upper gingivobuccal and transconjunctival incisions with lateral paracanthal extension. The mean interval from injury to operation was 16 months (range, 12 months to 4 years), and the mean follow-up was 1 year (range, 4 months to 3 years).

Results: Our surgical approach technique allowed excellent access to the infraorbital rim, orbital floor, zygomaticofrontal suture and anterior surface of the maxilla. Of the 14 patients, only 1 patient suffered a complication—oral wound dehiscence. Among the 6 patients who received infraorbital nerve decompression, numbness was gradually relieved in 4 patients. Two patients continued to experience persistent numbness.

Conclusion: Transconjunctival incision with lateral paracanthal extension combined with upper gingivobuccal sulcus incision offers excellent exposure of the zygoma-orbit complex, and could be a valid alternative to the bicoronal approach for osteotomy of malunioned zygoma.
\end{abstract}

Keywords: Conjunctiva / Eyelids / Osteotomy / Zygomatic fracture

\section{INTRODUCTION}

Severe posttraumatic facial deformities resulting from extensive facial fractures have long been challenging problems for the plastic surgeon. Delay in operative management secondary to combined life-threatening injuries, failure to appreciate the magnitude

\footnotetext{
Correspondence: Deok-Woo Kim

Department of Plastic and Reconstructive Surgery, Korea University Ansan Hospital, Korea University College of Medicine, 123, Jeokgeum-ro, Danwon-gu, Ansan 15355, Korea

E-mail: deokwookim@gmail.com

Received February 6, 2016 / Revised May 23, 2016 / Accepted May 23, 2016
}

of the initial facial injury, inadequate operative treatment, and operative complications contribute to their occurrence [1].

With more widespread adoption of craniofacial techniques, traditional approaches, such as repeated onlay bone grafts and alloplastic implants, have been relegated to secondary roles. Minor contour irregularities can be smoothed by grinding or onlay bone graft, but large onlay grafts are not recommended, especially in the malar area $[2,3]$. Restoration of normal anatomic position of displaced facial bones by appropriate osteotomies has become the standard of treatment for severe posttraumatic facial 
deformities.

In treatment of craniofacial and midfacial fractures, various approaches and designs of surgical incision have been described. Among them, bicoronal approach has been indicated for severe comminuted zygomatic arch and zygomatic bone fractures because it allows excellent access to the temporal and infratemporal spaces for management of severe traumatic injuries. Moreover, this is the approach of choice for delayed correction of major zygomatic deformities by osteotomy or extensive bone grafting [4]. However, there are many potential complications following a bicoronal incision, such as infection, hematoma, alopecia, visible scarring, and facial nerve injury [5]. Some surgeons advocate the lateral eyebrow approach for initial access to the zygomaticofrontal region [6], but it offers only a narrow operative field and may result in eyebrow scarring and hair loss [7]. Therefore, we have adopted a zygomaticofrontal suture osteotomy technique using transconjunctival incision with lateral paracanthal extension. We performed a retrospective review of clinical cases underwent correction of malunioned zygoma with the approach to evaluate outcomes following this method.

\section{METHODS}

Between June 2009 and September 2015, corrective osteotomies were performed in 14 patients with malunioned zygoma (Table 1). All operations were performed by a single surgeon. The mean age of patients was 48 years (range, 28-69 years). Eight patients were males, and six were females. Injury mechanisms included motor vehicle collision $(n=4)$, fall $(n=6)$, and assault $(n=4)$. The causes of the malunions are as follows: delayed operation due to life-threatening injuries such as intracranial hemorrhage $(n=8)$, missed diagnosis of fractures with non-intervention $(\mathrm{n}=2)$, and incomplete reduction/reconstruction at initial surgical intervention $(n=4)$. The mean interval from injury to operation was 16 months (range, 12 months to 4 years), and the mean follow-up was 1 year (range, 4 months to 3 years) (Table 1).

For preoperative assessment, all patients underwent clinical evaluations for bone integrity, position of the globe, change in canthal attachment, and soft tissue status. Radiographic evaluation (plain films, computed tomography [CT] scans) and ophthalmologic consultation were also performed [8].

Medical records were reviewed for detailed operation notes,

Table 1. Patient characteristics

\begin{tabular}{|c|c|c|c|c|c|c|c|c|c|c|}
\hline Patient & Sex & Age (yr) & Category & Site & $\begin{array}{l}\text { Injury } \\
\text { mechanism }\end{array}$ & Interval & Follow-up & Bone graft & $\begin{array}{c}\mathrm{ION} \\
\text { compression }\end{array}$ & Complementary operation \\
\hline 1 & Male & 54 & Dealayed operation & Left & Fall down & $1 \mathrm{yr}$ & $6 \mathrm{mo}$ & - & $(+)$ & $\begin{array}{l}\text { SMAS plication } \\
\text { contralateral face lift }\end{array}$ \\
\hline 2 & Male & 42 & Dealayed operation & Left & Fall down & $1 \mathrm{yr}$ & $1 \mathrm{yr}$ & - & - & - \\
\hline 3 & Male & 28 & Inadequate treatment & Both & $\begin{array}{l}\text { Motor vehicle } \\
\text { accident }\end{array}$ & $3 y r$ & $4 \mathrm{mo}$ & - & - & TFL sling, dermofat graft \\
\hline 4 & Female & 56 & Dealayed operation & Right & Assault & $1 \mathrm{yr}$ & $3 y r$ & - & $(+)$ & Dermofat graft, septoplasty \\
\hline 5 & Female & 54 & Inadequate treatment & Left & $\begin{array}{l}\text { Motor vehicle } \\
\text { accident }\end{array}$ & $4 \mathrm{yr}$ & $8 \mathrm{mo}$ & lliac bone & - & $\begin{array}{l}\text { Lateral canthopexy, fat } \\
\text { injection }\end{array}$ \\
\hline 6 & Female & 69 & Dealayed operation & Right & Fall down & $1 \mathrm{yr}$ & $1 \mathrm{yr}$ & - & $(+)$ & - \\
\hline 7 & Male & 43 & Dealayed operation & Left & Assault & $1 \mathrm{yr}$ & $1 \mathrm{yr}$ & - & $(+)$ & - \\
\hline 8 & Male & 56 & Dealayed operation & Right & Fall down & $1 \mathrm{yr}$ & $6 \mathrm{mo}$ & - & - & - \\
\hline 9 & Female & 46 & Dealayed operation & Left & Fall down & $14 \mathrm{mo}$ & $8 \mathrm{mo}$ & - & - & Upper blepharoplasty \\
\hline 10 & Female & 38 & Inadequate treatment & Left & Assault & $1 \mathrm{yr}$ & $2 \mathrm{yr}$ & - & $(+)$ & - \\
\hline 11 & Female & 61 & Dealayed operation & Right & $\begin{array}{l}\text { Motor vehicle } \\
\text { accident }\end{array}$ & $18 \mathrm{mo}$ & $1 \mathrm{yr}$ & - & - & - \\
\hline 12 & Male & 29 & Inadequate treatment & Right & Assault & $3 y r$ & $8 \mathrm{mo}$ & - & $(+)$ & - \\
\hline 13 & Male & 53 & Dealayed operation & Left & $\begin{array}{l}\text { Motor vehicle } \\
\text { accident }\end{array}$ & $2 y r$ & $1 \mathrm{yr}$ & - & - & Dermofat graft \\
\hline 14 & Male & 47 & Dealayed operation & Right & Fall down & $20 \mathrm{mo}$ & $6 \mathrm{mo}$ & - & - & - \\
\hline
\end{tabular}

ION, infraorbital nerve; SMAS, superficial musculo-aponeurotic system; TFL, tensor fascia lata. 
follow-up records, and perioperative photographs. Facial bone plain films and CT scans were analyzed for posttraumatic facial deformities. Among these, zygomatic arch views were used to measure degrees of correction (Fig. 1). In zygomatic arch view, the most anterior point of zygoma-orbit complex was used as a standard. A horizontal line was drawn from uppermost (anterior) point of normal side zygoma (Line A), and another line is drawn from that of malunioned zygoma (Line B). We measured the distance between the two lines before and after surgery for evaluating the degree of correction. In addition, to assess the horizontal width change, vertical line drawn from nasal septal bone was used as the base line. The line from the base line to the most lateral point of normal side zygoma denotes line $\mathrm{C}$, and the line from the base line to the most lateral point that of malunioned zygoma denotes line $\mathrm{D}$. We also measured the distance difference between the two lines before and after surgery for evaluating horizontal width change.

\section{Operative technique}

The procedure was performed under general anesthesia in the supine position. We operated on all patients using transconjunctival incisions with lateral paracanthal extension. After forced duction tests, cornea protectors were inserted into the eyes. The lower eyelid was everted, and 3 pairs of 5-0 Prolene sutures were placed just superior and inferior to the designed incision line, 2 to $3 \mathrm{~mm}$ beneath the inferior border of the tarsal plate. The conjunctiva was infiltrated with $2 \%$ lidocaine containing epinephrine 1:100,000. The conjunctiva and capsulopalpebral fascia were incised with a blade. After achieving hemostasis with bipolar cautery, a small amount of fatty tissue and orbital septum could be seen next to the capsulopalpebral fascia. The septum was incised with bipolar cautery or scissors, and the dissection plane was then exposed between the orbicularis oculi muscle and orbital septum. Preseptal dissection was performed with care. At the arcus marginalis, the periosteum on the orbital rim was incised and subperiorbital dissection was performed. For ease of dissection and wide exposure, lateral paracanthal incision preceded preseptal dissection if needed. The full thickness lower eyelid, involving the tarsal plate, was incised with scissors at a right angle at a point approximately 2 to $3 \mathrm{~mm}$ from the lateral canthus. The incision was then extended laterally parallel to the

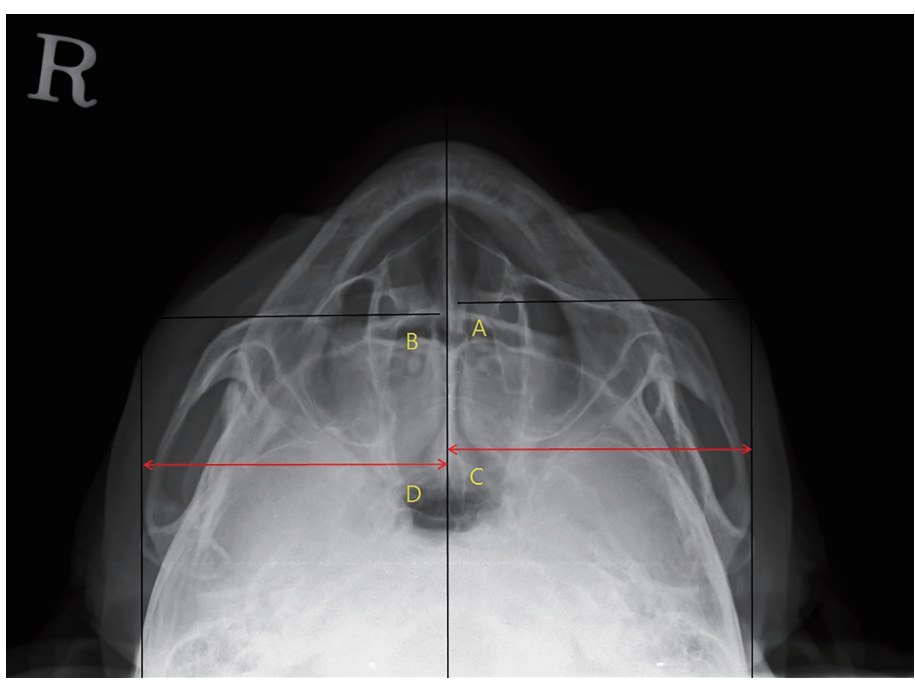

Fig. 1. Zygomatic arch view. Preoperative zygomatic arch view of a patient (No. 6) with right-sided zygomatic depression. The most anterior point of zygoma-orbit complex is used as standard. Line A denotes the normal position of the zygoma-orbit complex and line B the displaced position of the zygoma-orbit complex. The distance between line A and B was measured before and after surgery to assess height difference. Line C denotes the distance between base line (vertical line drawn from nasal septal bone used as standard) and most lateral point of normal side zygoma, and line D the distance from base line to that of malunioned zygoma. The distance difference between line $\mathrm{C}$ and $\mathrm{D}$ was measured before and after surgery to assess horizontal width change. 
natural skin crease. From this point, more subperiosteal dissection was necessary to reach the zygomaticofrontal suture area in which old fracture line could be found (Fig. 2) [8]. In the same way, the oral incision was placed approximately $5 \mathrm{~mm}$ from the gingivobuccal sulcus. Wide subperiosteal dissection proceeded superiorly to the infraorbital rim until the entire anterior face of the maxilla was exposed to minimize soft tissue adhesion. However, no separate procedure was performed for separating masticatory muscle from zygomatic bone complex.

Following the surgical exposure, the zygomaticofrontal suture was cut with a reciprocating saw. Then, the lateral orbital wall was cut with a round burr between the orbital surface of the zygomatic bone and the greater wing of the sphenoid to the anterior end of the inferior orbital fissure. The next step was to make a bone cut using an osteotome from the infraorbital rim just lateral to the infraorbital nerve extending down the anterior maxil- lary wall. Zygomatic osteotomy could follow the fracture lines of the original injury. Then, the reciprocating saw was introduced to the oral incision site, and the zygomaticomaxillary buttress was cut just lateral to the infraorbital foramen. The height difference across the osteotomies at the infraorbital rim and zygomaticofrontal suture were measured with a caliper before mobilization of the zygoma to assess the amount of correction. A Joker elevator was inserted behind the zygomatic bone and elevated with force to fracture the posterior attachment of the zygoma. In cases where the zygomatic arch fractures were unioned and not mobilized by the procedure, a curved osteotome was used on the zygomatic arch to complete the osteotomy. The zygoma was fixed into its new position with titanium miniplates (Fig. 3). The first plate was placed across the zygomaticofrontal suture or infraorbital rim. We chose the first site for fixation according to the degree of gapping. Fixation of zygomaticomaxillary buttress was
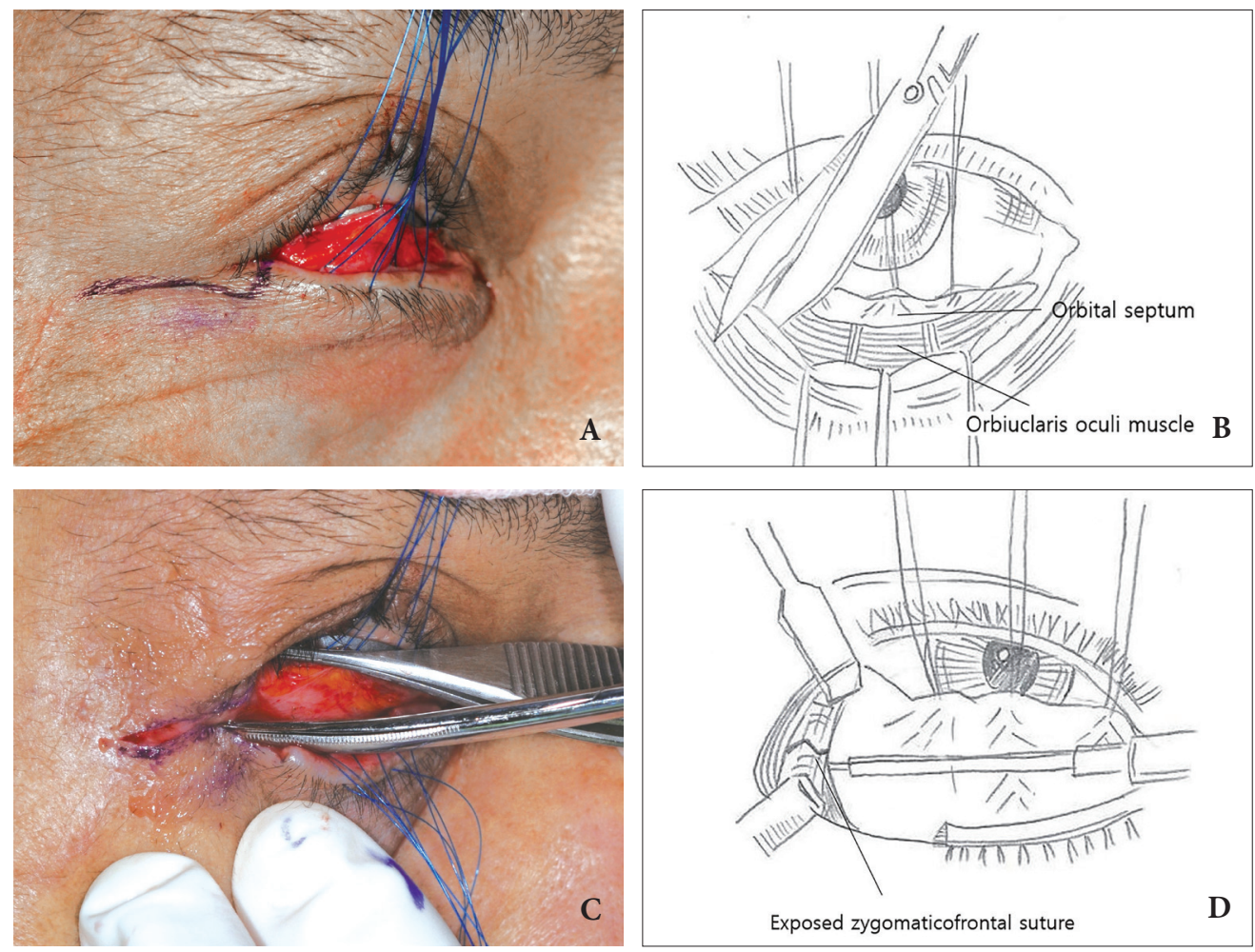

Fig. 2. Transconjunctival incision with lateral paracanthal extension. (A) Tagging sutures were placed and the conjunctival incision was made with a blade. The lateral paracanthal incision line was marked vertically at a right angle to the eyelid border at a point approximately 2-3 mm from the lateral canthus and was extended horizontally following the natural skin crease. (B) The full thickness lower eyelid incision was made vertically with tenotomy scissors. (C) The incision was extended laterally along the previous marking. (D) Dissection proceeded to reach the zygomaticofrontal suture. The zygomaticofrontal suture was exposed. 
done afterwards. If the zygomatic repositioning created an inferior orbit wall defect or the orbit had been widened by the previous fracture, we reconstructed the orbit using titanium reinforced polyethylene mesh (Synpor, DePuy Synthes Inc., Warsaw, IN, USA).

At the conclusion of surgery, the periosteum around the orbit was closed tightly with 4-0 Vicryl to prevent malpositioning of the lateral canthus and infraorbital soft tissue. The tarsal plate of the lateral paracanthal incision was repaired with 5-0 PDS (Ethicon, Inc., Somerville, NJ, USA), and the grey line of the eyelid border was repaired in an everted fashion to prevent notch deformity. The skin incision on the lateral canthus and lateral orbital rim was closed in a standard fashion. The conjunctiva was closed with continuous 6-0 Vicryl sutures, and the oral wound was closed tightly using fast absorbing Vicryl sutures.
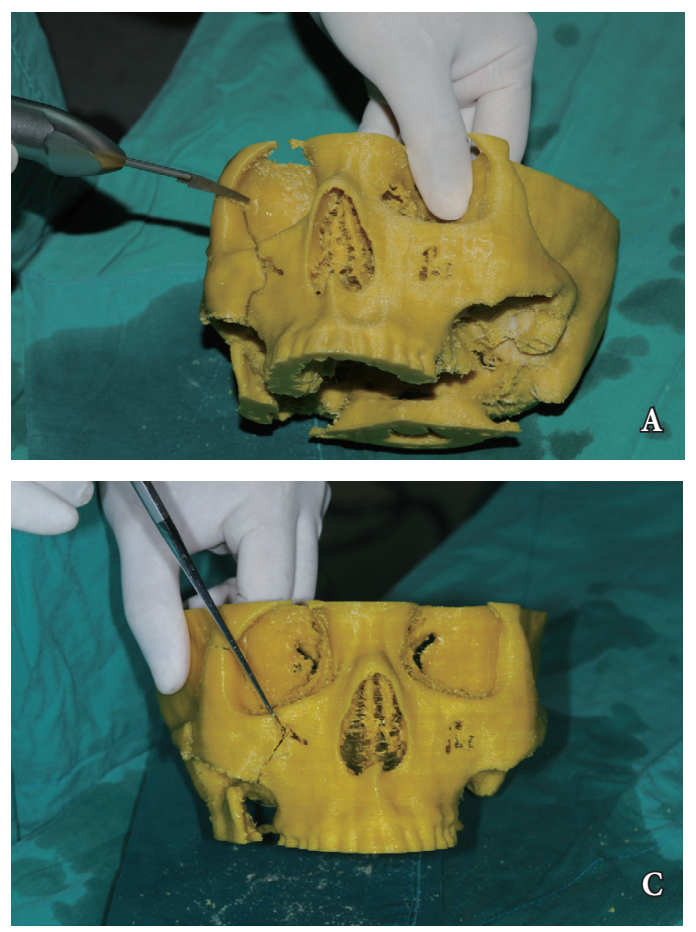

\section{RESULTS}

All patients received transconjunctival incisions with lateral paracanthal extension and gingivobuccal incision. Via the surgical incisions, we could easily access the infraorbital rim, orbital floor, zygomaticofrontal suture, and anterior surface of the maxilla. There was no need for coronal or supra-brow incisions. For 6 patients with symptoms of infraorbital numbness, nerve decompression was carried out by widening the infraorbital canal. Zygomaticomaxillary osteotomy was extended to the infraorbital foramen from the infraorbital rim and oral maxilla. The infraorbital canal was carefully explored, and bone spicules and canal edges were removed. Bone grafts to the malar area were performed in only one patient (No. 5), who had a history of communited zygomatic bone fracture (Table 1). Once skeletal foundations were reset, some ancillary procedures such as dermofat grafts and fat injection were performed in 6 pa-
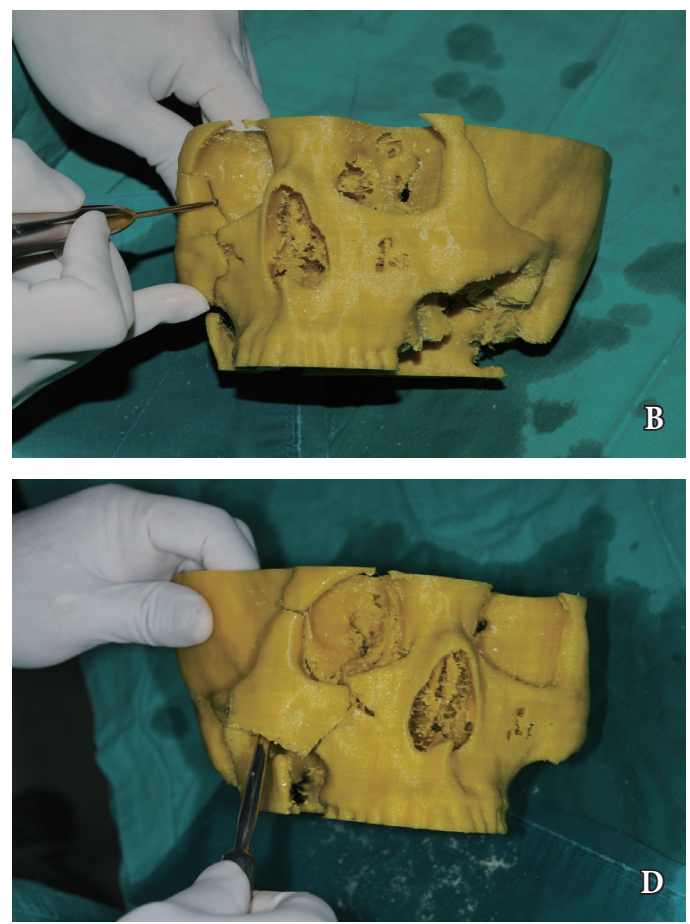

Fig. 3. Mock surgery for correction of malunioned zygoma. This skull model was made using a three-dimensional printing system. (A) The zygomaticofrontal suture was cut with a reciprocating saw. (B) The lateral orbital wall was cut with a round burr between the orbital surface of the zygomatic bone and greater wing of the sphenoid to the anterior end of the inferior orbital fissure. (C) A bone cut was made using an osteotome from the infraorbital rim just lateral to the infraorbital nerve extending down the anterior maxillary wall. A reciprocating saw was introduced to the oral incision site and the zygomaticomaxillary buttress was cut just lateral to the infraorbital foramen. (D) A curved osteotome was used on the zygomatic arch to complete the osteotomy. A Joker elevator was inserted behind the zygoma. The posterior attachment of the maxilla was easily fractured by gently elevating the zygomatic bone. 
tients as secondary operations. Among the six patients who received secondary operations, three patients underwent dermofat graft on nose to correct deformed nasal contour, and one patient received fat graft for correction of soft tissue atrophy on cheek and malar area. The average preoperative height difference in zygomatic arch view was $3.5 \mathrm{~mm}$ (range, 1.5 to $6.0 \mathrm{~mm}$ ), and the height difference had decreased to $0.6 \mathrm{~mm}$ (range, 0 to $2.0 \mathrm{~mm}$ ) after surgery. The average preoperative width difference in zygomatic arch view was $4.9 \mathrm{~mm}$ (range, 3.0 to $7.5 \mathrm{~mm}$ ), and the width difference was decreased to 0.4 $\mathrm{mm}$ (range, 0 to $2.0 \mathrm{~mm}$ ) after surgery, a reduction close to normal measurement value (Table 2). One patient (No. 3) experienced oral wound dehiscence with sinus abscess that was treated by wound irrigation and sinus mucosa stripping. Among the 6 patients who received nerve decompression, gradual improvement was observed in 4 patients, with two remaining patients continuing to experience numbness after 1 year (Table 2). The lateral paracanthal incision scars were inconspicuous, and none of the patients complained about the relatively well-hidden incision scar.

\section{Case 1}

A 69-year-old woman (No. 6) visited our clinic complaining of numbness on the right malar area. She had fallen 12 months before, but the fracture was not diagnosed at that time. A facial CT scan showed that the right zygoma was impacted medially. Corrective osteotomy and infraorbital nerve release were planned for the patient. The zygoma was approached via the upper gingivobuccal sulcus incision and conjunctival incision with lateral paracanthal extension. The old fracture line on the zygomaticofrontal suture and anterior maxillary wall was exposed. Some bony overgrowth observed around the infraorbital foramen. Osteotomies were made through the zygomaticofrontal suture, lateral orbital wall, infraorbital rim, and zygomaticomaxillary buttress (Fig. 4). The zygomatic bone was moved laterally, and the infraorbital canal was explored and widened to remove any possible compression on the nerve. The zygomatic bone was repositioned to its anatomical position and fixed with titanium plates. No complications were observed during the 12-month follow-up period.

\section{Case 2}

A 56-year-old man (No. 8) was admitted for correction of facial asymmetry. He had fallen from a 5-m height 12 months before and was cared for by the neurosurgeons, but the correction of fa-

Table 2. Postoperative outcome and complication

\begin{tabular}{|c|c|c|c|c|c|c|c|}
\hline Patient & Sex & Age (yr) & Postoperative follow-up & $\begin{array}{l}\text { Zygomatic arch view } \\
\text { (pre/post height } \\
\text { difference) }{ }^{\text {a) }}\end{array}$ & Complication & Sequelae & Other problem \\
\hline 1 & Male & 54 & Symmetry correction, sensory improved & $-4.5 /+1.0$ & - & - & - \\
\hline 2 & Male & 42 & Symmetry correction & $-3.0 /-1.5$ & - & - & - \\
\hline 3 & Male & 28 & Symmetry correction & $-2.5 / 0$ & Infection & - & - \\
\hline 4 & Female & 56 & Symmetry correction & $-5.0 /-2.0$ & - & $\begin{array}{l}\text { Consistent } \\
\text { numbness }\end{array}$ & $\begin{array}{l}\text { Post-traumatic stress } \\
\text { disorder }\end{array}$ \\
\hline 5 & Female & 54 & Symmetry correction & $-3.0 /-1.0$ & - & - & - \\
\hline 6 & Female & 69 & Symmetry correction, sensory improved & $-4.0 / 0$ & - & - & Glaucoma \\
\hline 7 & Male & 43 & Symmetry correction, sensory improved & $-5.5 /-1.5$ & - & $\begin{array}{l}\text { Consistent } \\
\text { numbness }\end{array}$ & - \\
\hline 8 & Male & 56 & Symmetry correction & $-6.0 /-2.0$ & - & - & Consistent headache \\
\hline 9 & Female & 46 & Symmetry correction & $-3.0 /-1.0$ & - & - & - \\
\hline 10 & Female & 38 & Symmetry correction, sensory improved & $-2.5 /-0.5$ & - & - & - \\
\hline 11 & Female & 61 & Symmetry correction & $-3.0 /+0.5$ & - & - & - \\
\hline 12 & Male & 29 & Symmetry correction, sensory improved & $-1.5 /+1.0$ & - & - & - \\
\hline 13 & Male & 53 & Symmetry correction & $-2.0 / 0$ & - & - & - \\
\hline 14 & Male & 47 & Symmetry correction & $-3.0 /-1.0$ & - & - & - \\
\hline
\end{tabular}

a)The most anterior point of zygoma-orbit complex compared with the normal side, $\mathrm{mm}$. 
cial bone fractures had been delayed because of a life-threatening cerebral hemorrhage. The patient had since recovered and wished for a more symmetrical face. In the operating room, we cut the zygomaticofrontal suture first with a reciprocating saw, and then the lateral orbital wall was cut with a round burr. We then performed an osteotomy from the infraorbital rim to the zygomatic buttress.
The zygoma was repositioned considering malar symmetry, and the findings of a mock surgery that had been performed earlier. Fixation of the zygomatic bone complex was conducted using 1.5 $\mathrm{mm}$ thick titanium miniplates. There was no inferior orbital wall defect and thus no need for obliteration. No complications were observed during the 6-month follow-up period. The patient was
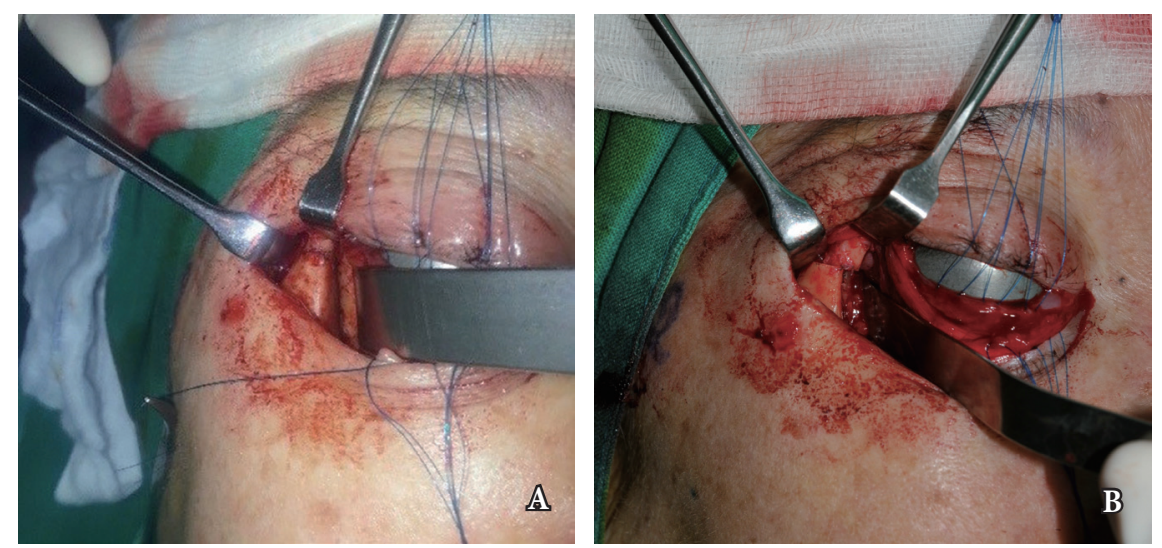

Fig. 4. Clinical photographs of case 1. (A) In the operative field, the old fracture line on the zygomaticofrontal suture was identified. (B) Following complete osteotomies around the zygomatic bone, the zygoma could be moved freely.
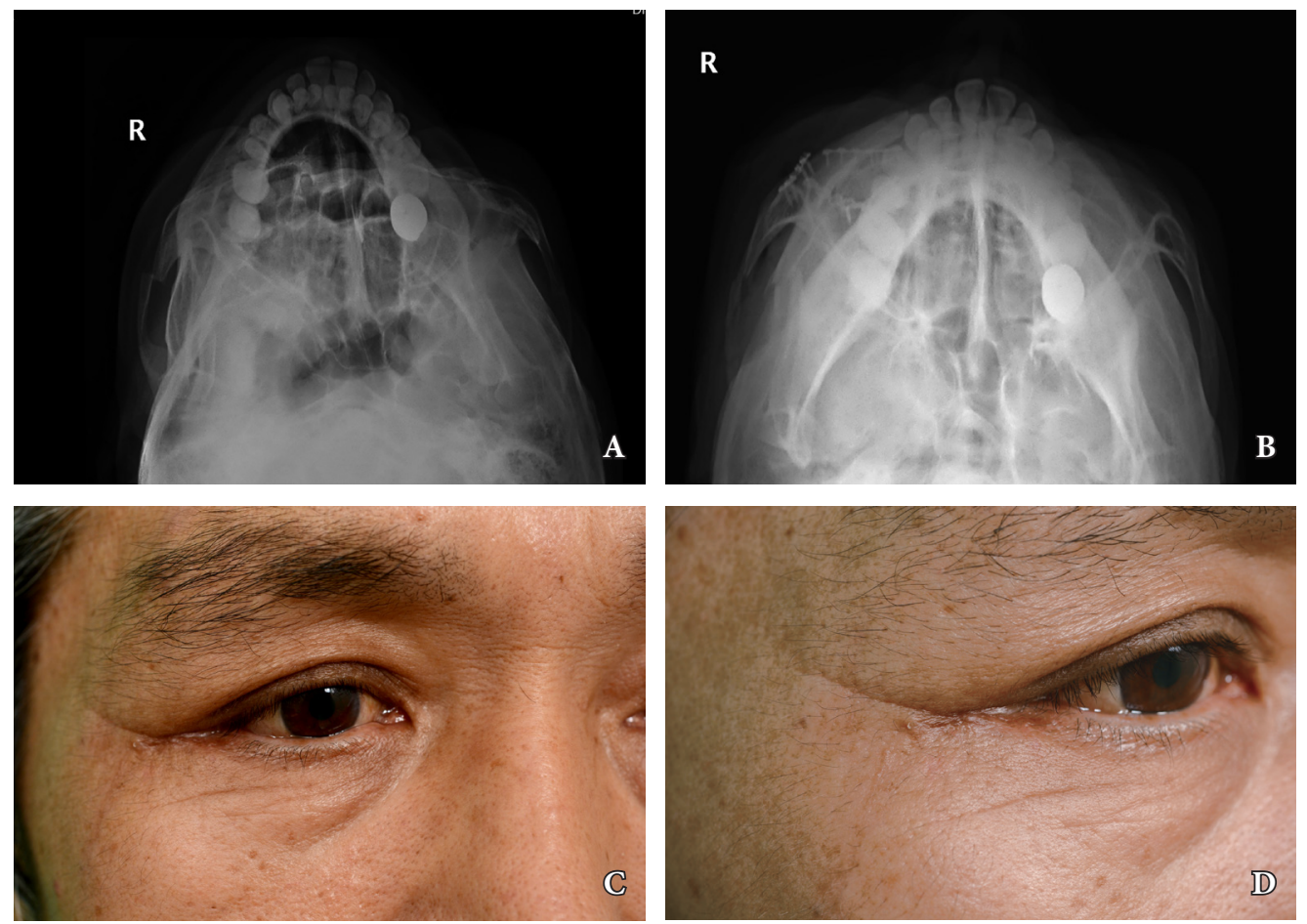

Fig. 5. Clinical photographs and radiographs of case 2. (A, B) Fracture displacement was identified in the preoperative zygomatic arch view plain film, and was corrected in the postoperative radiograph. (C, D) The lateral paracanthal incision healed well without scar formation 2 months postoperatively. The incision line was hidden by the natural skin crease. 
satisfied with the symmetry of his face (Fig. 5).

\section{DISCUSSION}

Complete regional exposure is usually required to achieve correction of malunioned zygoma. Traditionally, a bicoronal flap combined with a lower eyelid incision and an upper buccal sulcus incision has been used for exposure of the zygomaticofrontal suture area because this approach gives excellent exposure of the upper craniofacial skeleton [9]. Using the bicoronal approach, the calvarium, forehead, supraorbital rim, orbital roof and lateral orbital rim can be widely exposed. Reflection of the temporalis muscle enables access to the lateral wall of the orbit and temporal fossa. Converse et al. [10] noted that osteotomy for reduction of old fractures is an indication for the coronal approach in craniomaxillofacial trauma patients. Although a bicoronal approach can widely expose the lateral side of the zygoma-orbit complex, there are many potential complications. Coronal incisions can result in alopecia, and therefore, this incision should be carefully modified or not be used in patients with male pattern baldness. Other possible complications of the bicoronal approach include sensory disturbances of the supraorbital and preauricular areas, motor deficits of the frontal branches of the facial nerve, and hematomas under the flap [11].

Pozatek et al. [6] stated that "the lateral eyebrow approach is also applicable to treatment of old or malunioned zygoma fracture." This approach gives direct access to the zygomaticofrontal suture line. However, it may leave an objectionable scar and partial brow depilation. Moreover, the overextended incision can make damage to motor nerve branches to the orbicularis oculi [4].

Chuong and Kaban [7] advocated the use of upper eyelid incisions for initial access to zygomatic complex fractures. This approach offers greater versatility and enhanced accessibility to the superolateral rim compared to the lateral eyebrow approach. The soft tissues of the upper lid are thin and resilient, so the incision site can be easily maneuvered onto the bony surfaces. However, Langsdon et al. [12] reported that this approach was associated with a higher incidence of postoperative complications, such as eyelid-related complications and infection. Yanagisawa [13] suggested that this approach is not suitable for lateral or inferior displacements.
Subciliary incisions are also acceptable because it is desirable for exploration, sectioning of the orbital floor, accurate repositioning of the segment, and placement and stabilization of graft or implants. However, this approach can lead to visible scars and persistent edema, so careful attention to surgical detail can produce an aesthetic infraorbital scar line and little change for infraorbital nerve damage [4].

To the best of our knowledge, this article is the first examination of transconjunctival incision with paracanthal extension for corrective osteotomy of malunioned zygoma. With this approach, we were able to expose the zygomaticofrontal suture and sphenozygomatic suture sufficiently to perform osteotomy. Compared with previous methods, this procedure has many advantages $[14,15]$. First, our technique entails fewer skin incisions and minimal dissection, and can therefore reduce operation time, complication rate, and recovery time. Second, it produces inconspicuous scars and provides direct access to the zygomaticofrontal region for fracture reduction and plate fixation.

The lateral paracanthal incision technique, in which the lid is transected approximately 2 to $3 \mathrm{~mm}$ from the lateral canthus, does not disrupt the canthal angle and its attachment to the orbit, with less morbidity and better cosmetic outcomes than traditional lateral canthotomy. It also facilitates anatomical repair of the lid because of easy identification of the gray line, which serves as an important landmark [8]. In our series, there was no lid malpositioning, the most concerned complication of the lateral canthotomy.

As previously mentioned, the key to successful treatment of posttraumatic facial deformities is the establishment of a correct diagnosis and formulation of a well-organized treatment plan. The vertical, horizontal, and sagittal dimensions of the craniofacial skeleton should be reestablished first. Even if skeletal reconstruction itself is symmetric, there can be soft tissue problems that produce asymmetric and deformed facial contour.

Cohen et al. [1] noted that the general goals of posttraumatic facial deformity reconstructions are (1) to restore normal and anatomic bone alignment, (2) to reestablish the underlying skeletal support prior to addressing soft-tissue abnormalities, and (3) to replace missing tissue with like tissue. Perino et al. [4] stated that aesthetic derangements in the periorbital soft tissues occur secondary 
to bony displacement. Tessier [16] reported that several procedures, such as rhinoplasty, lateral canthopexy, and scar excision may be needed following reconstruction as secondary operations. In some of our cases, soft tissue augmentation procedures such as dermofat grafts or fat injections were needed after skeletal correction.

Prior to each operation, we practice mock practice operations using skull models (Fig. 3). Three-dimensional printing techniques combined with computed tomographic imaging were used to generate a precise skull model. With the aid of the threedimensional model and mock surgery, we were able to determine the exact osteotomy point and proper positioning of the zygomatic bone complex in our patients.

There are some limitations of this study. First, this study was retrospective. To address this limitation, all clinical information was verified in electronic medical records to ensure accuracy of clinical information. The small number of patients included is also a limitation. There is a possibility that unrecognized complications might be elucidated if sufficient numbers of patients were enrolled in the study. Finally, this study was case series study and did not compare across various reconstructive methods. Thus, there is a need for new prospective comparative studies evaluating the outcomes of correcting malunioned zygoma with various approaches. Despite these limitations, we believe that transconjunctival incision with lateral paracanthal extension combined with upper buccal incision is a valid alternative to the bicoronal approach for osteotomy of malunioned zygoma.

\section{REFERENCES}

1. Cohen SR, Kawamoto HK Jr. Analysis and results of treatment of established posttraumatic facial deformities. Plast Reconstr Surg
1992;90:574-84.

2. Hammer B, Prein J. Correction of post-traumatic orbital deformities: operative techniques and review of 26 patients. J Craniomaxillofac Surg 1995;23:81-90.

3. Freihofer PM, Borstlap WA. Reconstruction of the zygomatic area: a comparison between osteotomy and onlay techniques. J Craniomaxillofac Surg 1989;17:243-8.

4. Perino KE, Zide MF, Kinnebrew MC. Late treatment of malunited malar fractures. J Oral Maxillofac Surg 1984;42:20-34.

5. Abubaker AO, Sotereanos G, Patterson GT. Use of the coronal surgical incision for reconstruction of severe craniomaxillofacial injuries. J Oral Maxillofac Surg 1990;48:579-86.

6. Pozatek ZW, Kaban LB, Guralnick WC. Fractures of the zygomatic complex: an evaluation of surgical management with special emphasis on the eyebrow approach. J Oral Surg 1973;31:141-8.

7. Chuong R, Kaban LB. Fractures of the zygomatic complex. J Oral Maxillofac Surg 1986;44:283-8.

8. Kim DW, Choi SR, Park SH, Koo SH. Versatile use of extended transconjunctival approach for orbital reconstruction. Ann Plast Surg 2009;62:374-80.

9. Manson PN. Facial injuries. In: McCarthy JG, editor. Plastic surgery. Philadelphia: Saunders; 1990. p. 867-1141.

10. Converse JM, Byron S, Wood-Smith D. Malunited fractures of the orbit. In: Converse JM, McCarthy JG, Littler JW, editors. Reconstructive plastic surgery Philadelphia: W.B. Saunders; 1977. p. 1020-110.

11. Shepherd DE, Ward-Booth RP, Moos KF. The morbidity of bicoronal flaps in maxillofacial surgery. Br J Oral Maxillofac Surg 1985;23:1-8.

12. Langsdon PR, Rohman GT, Hixson R, Stumpe MR, Metzinger SE. Upper lid transconjunctival versus transcutaneous approach for fracture repair of the lateral orbital rim. Ann Plast Surg 2010;65:52-5.

13. Yanagisawa E. Symposium on maxillo-facial trauma: 3. pitfalls in the management of zygomatic fractures. Laryngoscope 1973;83:527-46.

14. Jones RH, Ching M. Intraoral zygomatic osteotomy for correction of malar deficiency. J Oral Maxillofac Surg 1995;53:483-5.

15. Lee KA, Kim JW, Kim YH, Sun H, Jung JH. Clinical extension of corrective malarplasty with tripod osteotomy. J Craniofac Surg 2012;23:693-5.

16. Tessier P. Total osteotomy of the middle third of the face for faciostenosis or for sequelae of Le Fort 3 fractures. Plast Reconstr Surg 1971;48:533-41. 